\title{
Evaluation of Measles Surveillance Systems in
}

\section{Ginnir District, Bale Zone, Southeast Ethiopia: A Concurrent Embedded Mixed Quantitative/ Qualitative Study}

This article was published in the following Dove Press journal:

Risk Management and Healthcare Policy

\author{
Falaho Sani Kalil (1D) \\ Mohammed Hasen Bedaso $\mathbb{I D}^{2}$ \\ Mohammed Seid Abdulle ${ }^{3}$ \\ Nuriya Umer Mohammed (iD ${ }^{4}$ \\ 'Negelle Borena Health Science College, \\ Negelle Borena, Oromia Regional State, \\ Ethiopia; ${ }^{2}$ Public Health Emergency \\ Management Department, East Bale \\ Zonal Health Office, Ginnir, Ethiopia; \\ ${ }^{3}$ Public Health Emergency Management \\ Department, West Harerghe Zonal \\ Health Office, Chiro, Ethiopia; ${ }^{4}$ Public \\ Health Emergency Management \\ Department, Oromia Regional Health \\ Bureau, Addis Ababa, Ethiopia
}

Background: Public health surveillance systems should be evaluated periodically to ensure the problems of public health importance are being monitored efficiently and effectively. Despite the widespread measles outbreak in Ginnir district of Ethiopia in 2019, an evaluation of measles surveillance systems has not been conducted. Therefore, we evaluated the performance of measles surveillance systems and key attributes in Ginnir district, Southeast Ethiopia.

Methods: We conducted a concurrent embedded mixed quantitative/qualitative study in August 2019 among 15 health facilities/study units in Ginnir district. The qualitative study involved a purposively selected 15 key-informants. Data were collected using updated guidelines for evaluating surveillance systems based on CDC's Framework.

Results: Records of 15 study units were reviewed and 15 key informants participated. The structure of surveillance data flow was from the community to the respective upper level. Emergency preparedness and a response plan was available only at the district level. Weekly report completeness and timeliness were $95 \%$ and $87 \%$ respectively. We found weak supportive supervision and feedback, and no regular analysis and interpretations of surveillance data. The participation of surveillance stakeholders in implementation of the system was good. The surveillance system was found to be useful, easy to implement, representative, and can accommodate and adapt to changing operating conditions. Report documentation and quality of data was poor at lower level health facilities. The stability of the system has been challenged by a shortage of budget, logistics, staff turnover and lack of update trainings.

Conclusion: The surveillance system was acceptable, useful, simple, flexible, and representative. Quality of data, timeliness, and the stability of the system were attributes that require improvement. The overall performance of measles surveillance systems in prevention and control of measles was weak. Hence, regular analysis of data, preparation, and dissemination of epidemiological bulletin, capacity building, and regular supervision and feedback are recommended to enhance performance of the system.

Keywords: surveillance system evaluation, measles, Ginnir district, Bale zone, Ethiopia

\section{Background}

Public health surveillance is the continuous and systematic collection, analysis, interpretation, and dissemination of data regarding diseases or health-related events that have significant public health importance, and is used in public health action. ${ }^{1}$
Negelle Borena Health Science College Negelle Borana, 186, Oromia Regional

State, Ethiopia

Tel +251913963292

Email falahsani@gmail.com
Risk Management and Healthcare Policy 2021:14 997-1008

submit your manuscript DovePress if in $>$

http://doi.org/10.2147/RMHPS295809 
The purpose of evaluating public health surveillance systems is to monitor the efficiency and effectiveness of the system and provide recommendations for improving the quality. ${ }^{1-3}$ Public health surveillance systems have been developed to address a range of public health needs and include a variety of data sources essential to public health action. ${ }^{1,4}$ The data disseminated by a public health surveillance system are useful for immediate public health action, to measure the burden of diseases, to monitor trends, detection of outbreaks, planning the allocation of resources, implementation, and evaluation of control programs. $^{2-4}$ Public health surveillance is carried out through a system in the health sector extending from the central level down to the peripheral health facilities, and the upward reporting and downward feedback mechanism through sets of communication channels. ${ }^{1,5}$

To ensure rapid detection of public health threats and prompt response to and recovery from various public health emergencies, the Ethiopian Public Health Institute (EPHI) designed a fully integrated, all-hazards, adaptable and all health approach national preparedness and response system, called the Public Health Emergency Management (PHEM) system. ${ }^{5}$ PHEM is the process of emergency preparedness, early detection, response, and recovery from the consequences of public health threats to minimize the health and economic impacts from emergencies. Building a strong PHEM and strengthening its capacity will ensure the proper implementation of the 2005 International Health Regulation (IHR). Nationally, 23 priority diseases that should be reported immediately and on a weekly bases were selected and included into the routine surveillance system. Of these, measles is one of the immediately reportable priority diseases which has high epidemic potential. $^{5}$

Measles is an endemic disease in Ethiopia with outbreaks reported every year. ${ }^{6}$ A total of 7,445 confirmed cases of measles were reported from Ethiopia between 2016 and $2018 .^{7}$ In the year 2019 , a total of 8,202 suspected measles cases were reported from Oromia, Amhara, Afar, and Somali regions of Ethiopia. ${ }^{8}$ Furthermore, there was a widespread outbreak of measles which affected 1,043 people, with five deaths in Ginnir district of Bale zone in 2019, and the outbreak lasted for 5 months. ${ }^{9}$

The overall purpose of surveillance is to monitor the trends over time, detection of the occurrence of the diseases, identify the deviation from the threshold at the earliest point, and provide a prompt response. Furthermore, as an early warning system, it guides prevention of the disease before the occurrence of outbreak. ${ }^{5,10}$ To ensure that the public surveillance system is meeting its objective, its evaluation should involve an assessment of system attributes, including simplicity, level of usefulness, flexibility, data quality, acceptability, predictive value positive, representativeness, timeliness, and stability. ${ }^{1}$

Thus, the public health surveillance system should be evaluated periodically to determine how well the system is operating. ${ }^{1,10}$ However, the data on performance of the surveillance system are very limited in the country, particularly in the study area. Moreover, despite a widespread outbreak of measles in Ginnir district in 2019, the performance of the surveillance system was not evaluated. Therefore, the focus of this study was to evaluate the performance of a measles surveillance system and its key attributes in Ginnir district, Bale zone, Southeast Ethiopia.

\section{Methods \\ Study Setting}

The study was conducted in Ginnir district of Bale zone, which is located at a distance of $122 \mathrm{~km}$ from Robe town, the capital of Bale zone, and $557 \mathrm{~km}$ to the Southeast of Addis Ababa, the capital city of Ethiopia. Based on the 2007 national census projection, the estimated population of the district was 164,702 in 2019. Of these, 83,998 (51\%) were females. ${ }^{11}$ Administratively, the district has 32 rural kebeles (the lowest administrative unit within the district). There are eight health centers and 32 health posts in the district that provide primary healthcare services to the community, including public health emergency management. No private health facility is available in the district. At the district level, the surveillance activities are coordinated by a PHEM officer who is responsible for organizing the implementation of surveillance activities in the health facilities under the district catchment. Similarly, there are PHEM focal persons assigned at health centers and health posts for organizing the surveillance activities, preparation, and submission of weekly reports to the district health office.

\section{Study Design and Period}

We conducted a concurrent embedded mixed study approach of both quantitative and qualitative methods in August 2019, using 2018 as the base year. A crosssectional study was carried out to determine the performance of a surveillance system in the district. The 
qualitative interview was carried out with PHEM focal of the district health office and health facilities.

\section{Sample Size and Sampling Procedure}

A total of 15 study units/sites were included in the study. We included the district health office, and out of eight health centers in the district, four $(50 \%)$ were selected using lottery method. There are 32 health posts in the district, which is four satellite health posts under each health center catchment. Two health posts from the two cluster health centers, and three from the remaining two cluster health centers were selected using lottery method (Figure 1). A total of four health centers and 10 health posts were included in the study. For the qualitative interview, a purposive sampling technique was employed to select the key informants. The key informants were the district PHEM officer, the four health centers PHEM focal person, and ten Health Extension Workers (HEWs) from the health posts. As a result, the qualitative part involved 15 key informants inthe study. At the health post level, where surveillance activities were implemented by at least two health extension workers, we interviewed the most senior HEWs, considering them as a rich source of information.

\section{Data Collection Tools and Techniques}

This study evaluates the measles surveillance system using CDC framework, updated guidelines for evaluating public health surveillance systems. ${ }^{1}$ A semi-structured questionnaire
(Supplementary material) was developed using the checklist for evaluating public health surveillance systems. ${ }^{1}$ The data were collected through record reviews, document observation, and face-to-face interview of key stakeholders. For the quantitative survey, the source of data were surveillance reporting formats, records, and documents in the district health office and health facilities. For the qualitative study, the key informants such as district PHEM officer, health centers PHEM focal personnel, and health extension workers from health posts were interviewed using a semi-structured interview guide with flexible probing technique. Data regarding the availability of surveillance guidelines, reporting formats and documentation, registration of cases, completeness and timeliness of report, and the quality of data were assessed by quantitative survey by examining report format, records, and documents. Information concerning the operation of surveillance and reporting system, case detection, and attributes such as simplicity, flexibility, predictive value positive, representativeness, and stability of the system were explored by the key informants. Information concerning the analysis and interpretation of surveillance data, epidemic preparedness and management, outbreak investigation, supportive supervision and feedback, and the acceptability of surveillance system were assessed both by document observation and explored by key informants.

Data were collected by two field epidemiologists under the supervision of principal investigator. Interviews of the participants were continued until saturation was reached, meaning the investigator agreed that there was redundancy in the responses and no new idea emerging.

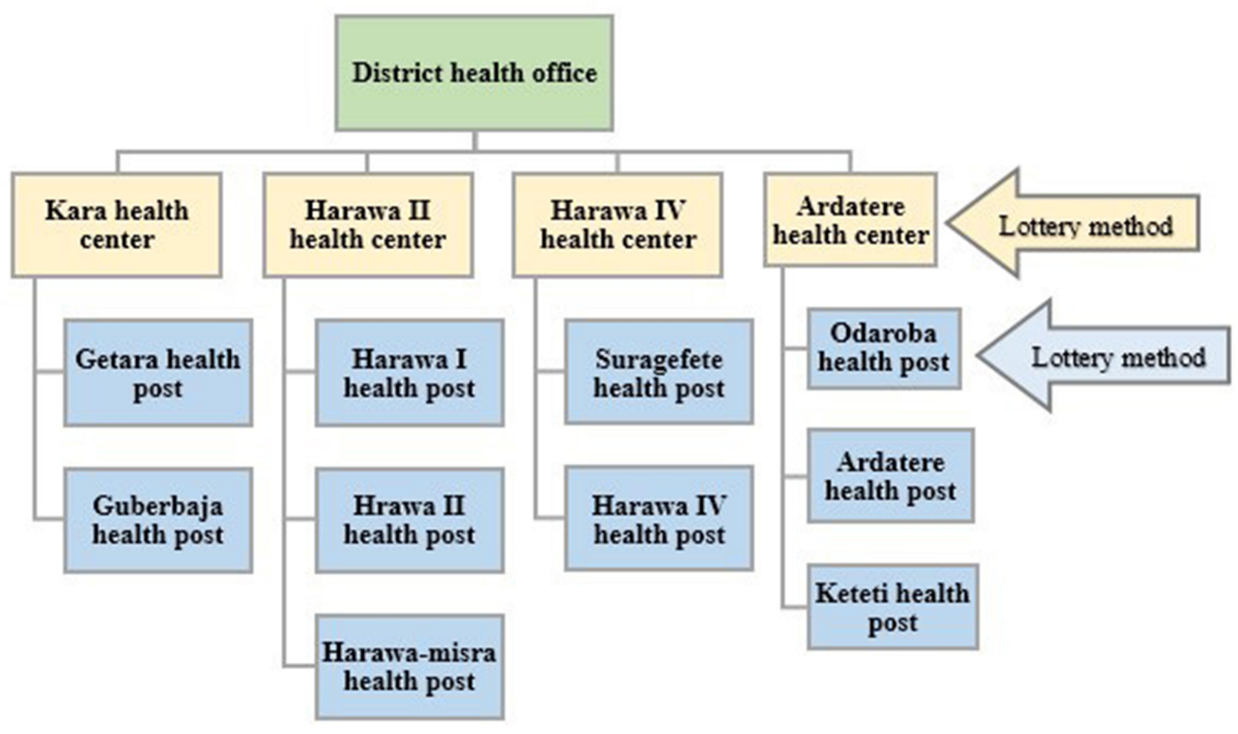

Figure I Schematic presentation of sampling procedure for evaluation of measles surveillance systems in Ginnir district, Southeast Ethiopia, 2018. 


\section{Data Quality Assurance}

A two days training was provided to the data collectors on the overall data collection procedure. The reports and records obtained from the health facilities were cross-che cked with the documented data at the district health office to check the consistency and accuracy of the data. During the session of each visit, we briefed the stakeholders about the purpose of the assessment, which was to evaluate the performance of the system rather than individual performances. Prior to data collection, discussion was made with stakeholders from Bale zonal health department, Ginnir district health office, and the health facilities, to ensure evaluation of the system addresses appropriate questions and attributes, to produce useful and acceptable findings.

\section{Data Processing and Analysis}

The quantitative data were entered and analyzed using Microsoft Excel 2016. All questionnaire responses were dichotomized except for the open-ended questions. Quantitative findings were summarized by their frequency and proportion. Qualitative data were analyzed manually using thematic analysis. Data were cleaned before analysis and the qualitative findings were narrated and summarized based on thematic areas to supplement the quantitative results.

\section{Operational Definitions}

\section{Usefulness}

A public health surveillance system is useful if it contributes to the prevention and control of adverse healthrelated events, including an improved understanding of the public health implications of such events. The level of usefulness was measured by the actions taken as a result of analysis and interpretation of the data from the public health surveillance system, the system useful to detect the diseases and outbreaks, providing estimates of the magnitude of morbidity and mortality. ${ }^{1}$

\section{Completeness}

The proportion of health facilities that submitted a weekly report to the higher level, out of the expected facilities in the catchment area (district, PHCU, kebele). ${ }^{1}$

\section{Timeliness}

The timeliness of the report was assessed at two levels. First, it was calculated by assessing how many of its expected reports were submitted to the next level within the prescribed time. The report is timely for the health facilities if a weekly report is submitted to the district health office every Monday before midday, and a timely report for the district is if the district health office submitted the compiled report to the zonal health department every Tuesday before midday. Second, the time interval between the onset of a health-related event and the reporting of the event, and the time required for the identification of an outbreak and initiation of control and prevention measures. ${ }^{1}$

\section{Simplicity}

Refers to the structure of the system and the ease of implementation while still meeting its objectives. ${ }^{1}$ In this study, the simplicity of the surveillance system was measured in terms of clear and easily understandable case definition, route of data flow, difficulty in completing surveillance data, and time taken to complete surveillance data.

\section{Flexibility}

The ability of the system to adapt to changing information needs or operating conditions with little additional time, personnel, or allocated funds. ${ }^{1}$ In this study, the flexibility of the surveillance system was assessed in terms of accommodating change in the existing procedure, a revised case definition, additional data sources, personnel, case detection, and reporting format.

\section{Data Quality}

Data quality was assessed based on content completeness of the reporting formats and validity of the data recorded. ${ }^{1}$ The fields examined for completeness include the number of cases and deaths, the date the report was sent and received, and the blank responses. Validity of recorded data at health facilities compared to the reported data at the district level.

\section{Acceptability}

Willingness of surveillance stakeholders to implement the system as expressed by their active participation in case detection and reporting. ${ }^{1}$ Acceptability was measured by completeness of report forms, timeliness of data reporting, and use of standard case definition.

\section{Predictive Value Positive}

The proportion of cases detected by the surveillance case definition that actually have the disease. ${ }^{1}$

\section{Representativeness}

Measured in terms of distribution of a health-related event by time, place, and person, the health service coverage, and the reporting of surveillance data from all health facilities. ${ }^{1}$ 


\section{Stability}

Refers to the reliability (ie, the ability to collect, manage, and provide data properly without failure) and availability of the public health surveillance system which is the ability to be operational when it is needed. ${ }^{1}$ Measured by the availability of a surveillance focal person at all levels and integration of the system to routine healthcare delivery.

\section{Results}

Report formats and records in the district health office, four health centers, and ten health posts were reviewed. Fifteen health workers participated and were interviewed in this study. Eleven (73.3\%) of these were female. Out of 15 key informants, five $(33.3 \%)$ were Public Health professionals, while $10(66.7 \%)$ were Health Extension Workers. The median age of the health workers was 34 years $(\mathrm{Q} 1=31.5 ; \mathrm{Q} 3=36.5)$ and the median duration of service provided was 9 years $(\mathrm{Q} 1=8 ; \mathrm{Q} 3=11.3)$. The population under surveillance were the population of Ginnir district, estimated to be 164,702 . Of these, $83,998(51 \%)$ were females and all are rural residents.

\section{Operation of Surveillance,}

\section{Communication, and Reporting System}

The routine flow of surveillance data is usually from reporting sites to the next level up to the central level. The health posts prepare and send their weekly surveillance report to the cluster health centers every Monday in the morning via a phone call. On the same days, in the afternoon, the health centers in turn aggregate the surveillance report received from the catchment health posts and send to the Ginnir district health office. Similarly, the district health office receives reports from the health centers using phone calls and sends to Bale Zonal Health Department on Tuesday afternoon (Figure 2). The surveillance system is fully integrated into the routine healthcare delivery system and implemented throughout the year without interruption. The community and health facilities, especially health posts, are the main source of information. The report submission to the higher level was through a phone call, followed by the paper-based report submission. All four health centers and ten health posts has no wired phone and they communicate with the next level with their mobile phone. The district health office has a wired office phone and Wi-Fi network. As represented in Figure 2, the flow of surveillance data and information

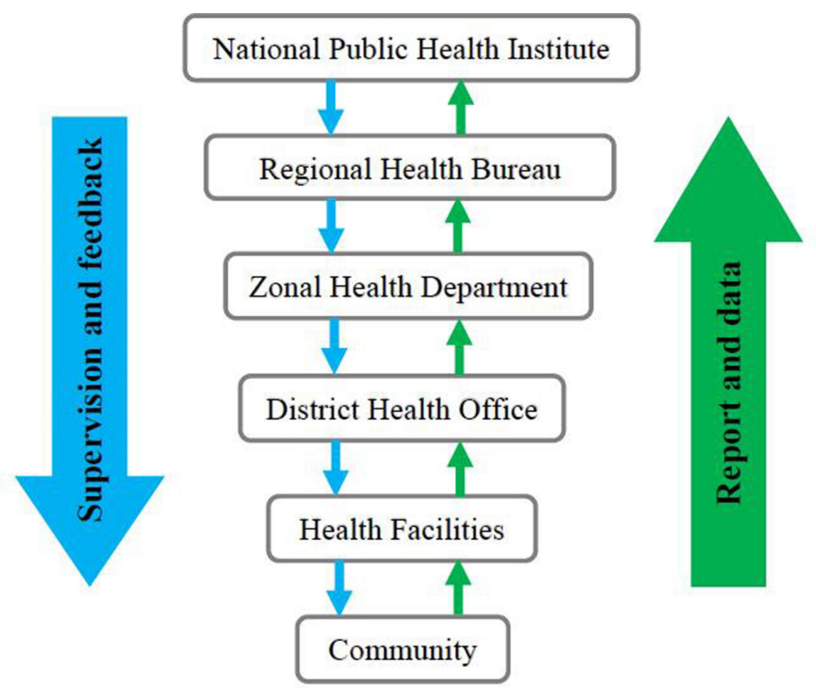

Figure 2 Diagram showing the flow of surveillance data and feedback in Ginnir district, Southeast Ethiopia, 2018.

is from the community to a higher level, whereas supervision and feedback follow the reverse direction.

\section{Availability of Surveillance Guidelines, Reporting Formats, and Documentation}

The national PHEM guideline was available at the district level and in all visited health centers. However, out of ten health posts, only three $(30 \%)$ of the visited health posts had a national PHEM guideline manual. There was a shortage of standard weekly reporting format in the visited health centers and health posts. Out of four health centers and ten health posts, the weekly reporting format was available in two $(50 \%)$ health centers and eight $(80 \%)$ health posts. In addition, the case-based reporting format was also not available in the visited health facilities. However, the weekly surveillance reporting format and measles case-based reporting format is available at Ginnir district health office. Regarding the documentation of a weekly surveillance report, all health centers and the district health office had a copy of each week report in the file cabinet. However, a copy of weekly surveillance report is not available at the visited health posts.

\section{Case Detection and Registration}

The case definition for measles disease is available at the district health office and all health facilities. The PHEM focal person of the district health office and visited health centers fully understood the measles case definition. However, four $(40 \%)$ of the health extension workers at the health post understood the measles case definition. The 
measles cases definition was posted on a wall or notice board of all health centers and three (30\%) of the health posts. Healthcare workers have been detecting suspected cases of measles using standard case definitions. All visited health centers and only three $(30 \%)$ of the health posts were using a clinical register to record the priority reportable diseases including measles. In addition, no rumor registration logbook was available at the visited health facilities.

\section{Analysis and Interpretation of Surveillance Data}

All health centers had at least one computer in addition to the desktop computer for the health management information system. However, none of the health centers used the computer for the analysis of surveillance data. The health centers send their weekly surveillance report which includes only the total number of cases and performed activities to the district health office, without analysis or interpretation of the data. The district health office and all health facilities had a denominator which helps to describe the surveillance data epidemiologically in terms of person, place, and time, such as the total number of populations disaggregated by age group, sex, and kebeles, etc. However, the district health office simply aggregates the reports received from the health centers and sends to the zonal health department without performing analysis of the surveillance data. In addition, there is no monitoring of accumulation of susceptible individuals for measles diseases over time using the measles risk assessment tool by the district health office, even if data on vaccination coverage and annual birth cohort is available.

\section{Epidemic Preparedness and Management}

Ginnir district health office has an Emergency Preparedness and Response Plan (EPRP), but there is no allocated budget to respond in case an emergency occurred. The health facilities did not have a written EPRP and outbreak investigation checklist. Stocks of the emergency drug and supplies for epidemic response were not available either at the district health office or health facilities, however whenever an outbreak existed, the health centers mobilize drugs and other medical supplies from their store which was reserved from the routine services. In addition, the district health office mobilizes emergency drug and supplies from the zonal health department every time an outbreak occurred. The district was established by a Rapid Response Team (RRT) and Multisectoral PHEM task force (Epidemic Management Committee) which is coordinated by district chief administrator; however, they are activated only when the outbreak exists. There are no documented meeting minutes by the committee. There was no vehicle assigned specifically to the public health emergency management department at the district level. The PHEM focal person from the district health office and health centers had received training on basic PHEM in the year 2018. However, out of 10 health extension workers interviewed, only two (20\%) of them received basic PHEM training.

\section{Outbreak Investigation}

In 2019, there was a widespread measles outbreak in Ginnir district which affected 1,043 people, with five deaths. According to the district PHEM focal, the outbreak lasted for 5 months and affected all the 32 kebeles in the district. A sample of five suspected cases were collected and confirmed by laboratory investigation at the national public health institute. The district encountered a shortage of emergency drugs and supplies during the outbreak. The cases were recorded on the line list and analyzed by time, place, and person according to the district PHEM focal. However, there was no documented report or standard procedure available for investigation of the measles outbreak. In addition, there was no assessment of the lessons learnt from the outbreak or post-epidemic evaluation conducted by the epidemic management committee to prevent the occurrences of outbreaks in the future.

\section{Supportive Supervision and Feedback}

Even though the district health office and health centers had a supervision plan, the supportive supervision was not conducted regularly. All study sites did not have a supervision checklist to assess PHEM activities including measles surveillance in detail. However, there was an integrated checklist which includes only a few data elements regarding PHEM at the district health office and health center. The district PHEM department had been supervised by zonal once in the year, but no written feedback of supervision was provided to the district. The Ginnir districts PHEM department had conducted two supportive supervisions to the health centers on integrated activities including surveillance. Out of ten health posts, only six $(60 \%)$ were supervised by the health centers concerning the surveillance activities in 2018. Despite this, there was no written letter of feedback provided to 


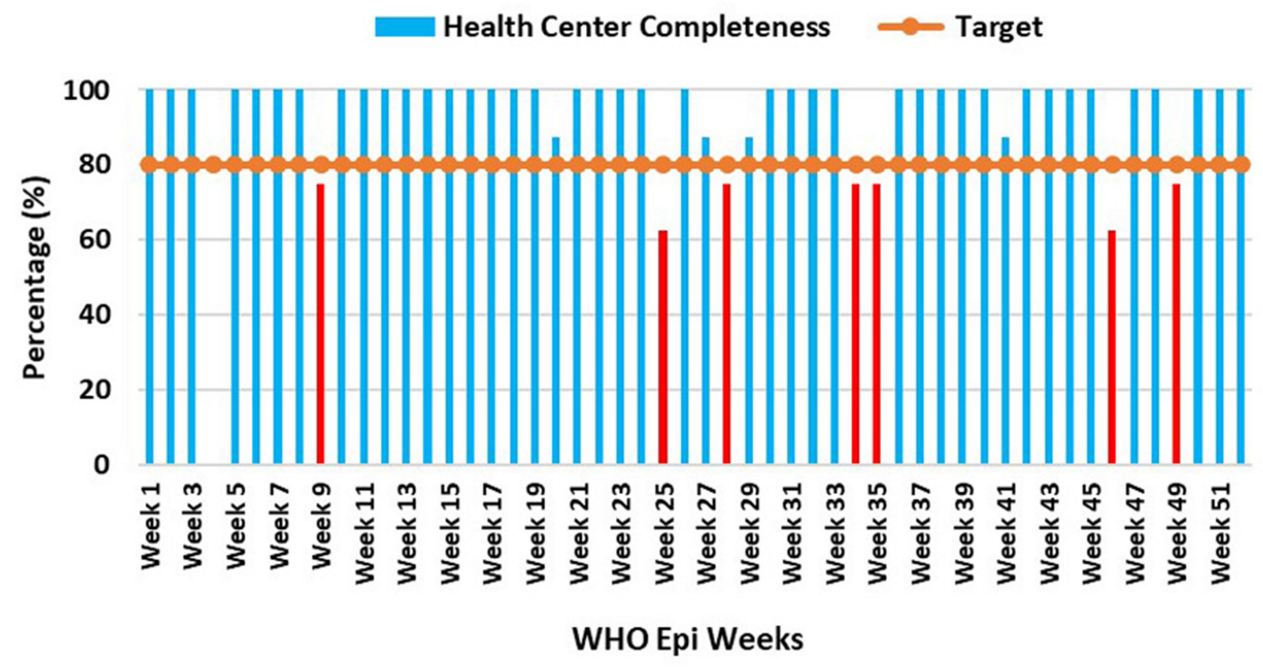

Figure 3 Completeness of health centers weekly surveillance report by WHO weeks, Ginnir district, Southeast Ethiopia, 2018.

the district PHEM department and health facilities from the respective higher level. The common reason for poor supervision activities were, shortage of manpower, lack of resources, poor coordination, and work overload according to the PHEM personnel. The district PHEM department and health centers provides only verbal feedback during quarterly and monthly meetings. In general, there was no regular supervision and feedback system, weekly bulletin preparation, or dissemination regarding public health surveillance in the district and health facilities.

\section{Attributes of Surveillance System Level of Usefulness}

According to the respondents, the surveillance system is very helpful to determine the magnitude of morbidity and mortality of diseases under surveillance as well as to assess the effectiveness of prevention and control measures for the priority diseases including measles. For the surveillance officers, the system helped to detect outbreaks early and take action to prevent epidemics. The system also helps to identify the priority areas for each disease under surveillance and for resource allocation. However, there are no actions taken as a result of analysis and interpretation of the data from the public health surveillance system.

\section{Completeness}

The completeness of surveillance report varies from one health facility to another. The report completeness by the health centers in Ginnir district was below the expected level of $80 \%$ in the WHO week $4,9,25,28,34,35,46$, and 49 in 2018 as shown in Figure 3. However, the average annual report completeness by the health centers was $93 \%$ in 2018 . The report completeness by the health posts were below expected level of $80 \%$ in the WHO week $4,25,35$, and 39, however, the average annual report completeness was $95 \%$ (Figure 4). Furthermore, the average completeness of surveillance report by the district was 95\% in 2018, as presented in Figure 5.

\section{Timeliness}

In the visited health facilities and at the district level, the date the report was received was not recorded on the report, as a result it was difficult to calculate the report timeliness. However, the average surveillance report timeliness calculated by the Ginnir district health office in 2018 was $87 \%$. Another aspect of timeliness is the time required for the identification of outbreaks or the effect of control and prevention measures, and the time interval between the onset of health-related events and the reporting of the event. According to the district PHEM officer, the recent measles outbreak which occurred in 2019 was reported to the zonal health department after 2 weeks of confirmation by the district. The intervention for control of the outbreak was also initiated by the district after 1 month of confirmation due to a shortage of resources. Additionally, the outbreak response, immunization targeting children aged 6 months to 14 years, was also initiated after 2 months.

\section{Simplicity}

All key informants agreed that measles case definitions for identification of the suspected cases are clear and easy to 


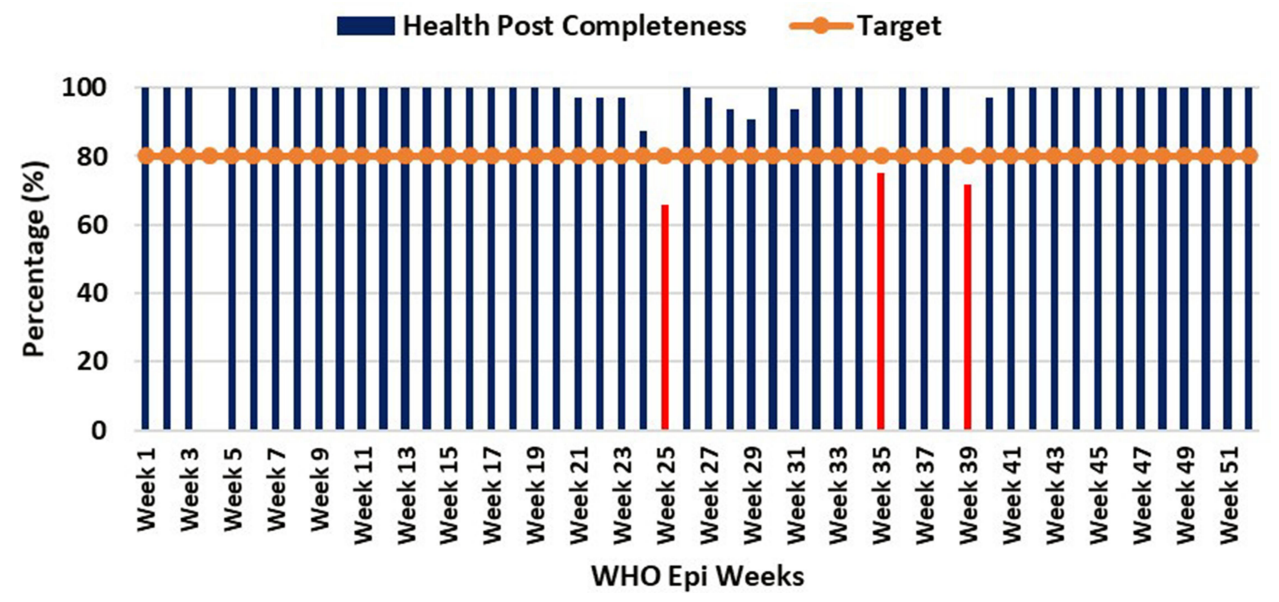

Figure 4 Completeness of health posts weekly surveillance report by WHO weeks, Ginnir district, Southeast Ethiopia, 2018.

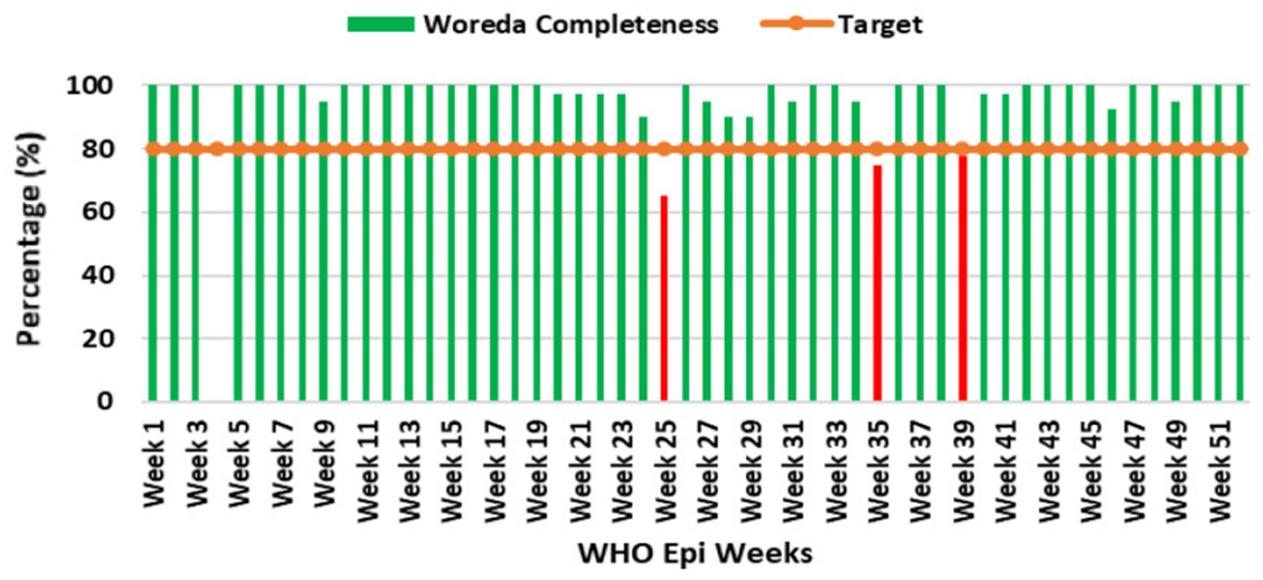

Figure 5 Overall completeness of weekly surveillance report by WHO weeks in Ginnir district, Southeast Ethiopia, 2018.

understand. Measles standard case definition was mostly used at the health center level, while a community case definition which is simplified and translated to the local language Afan Oromo is used by the health extension workers at the community level. All stakeholders stated that the route of surveillance data flow is clear and they are familiar with when and to whom the report was sent. The surveillance reporting formats are also easy to complete the data and also well understood by all respondents including health extension workers. The time to fill the weekly surveillance report is 20-25 minutes by the district PHEM officer, while it takes only 15-20 minutes by health centers PHEM focal and health extension workers.

\section{Flexibility}

The district PHEM officer and health facilities respondents agreed that the current weekly surveillance reporting format can accommodate new health events. They also explained that implementation of national PHEM guideline will not be difficult with changes in the existing procedure, personnel, case detection, and report forms.

\section{Data Quality}

Even though the majority of the fields in the surveillance reporting format were correctly filled, we have observed the following gaps related to the data quality; blank spaces that should be filled with zero (0) number were not recorded, the starting and ending dates of the week were also not completely filled. In addition, the date the report was received and sent is an important variable to determine the timeliness of the reporting, but commonly missed at the district and health facility level.

\section{Acceptability}

The surveillance officials have been actively participating in reporting surveillance data, as indicated by $95 \%, 93 \%$, 
and $95 \%$ achievement in the average report completeness by the district health office, health centers, and health posts, respectively. All health centers and health posts have been using the standard measles case definition for the detection of suspected cases, and appropriately using the reporting format. Hence, the surveillance system was acceptable by district PHEM officer, health centers PHEM focal persons, and health extension workers.

\section{Predictive Value Positive}

Calculating the predictive value positive (PVP) of measles requires the records of information obtained from the public health surveillance system. A record of the number of suspected measles cases detected by the surveillance system and those confirmed to have measles by the laboratory investigation of Immunoglobulin M (IgM) is required to calculate the PVP for measles surveillance. As a result of incomplete documentation of this data at district and health facility level, it was difficult to calculate PPV. However, during the measles outbreak in 2019, out of the collected five samples from the suspected measles cases and tested at the national laboratory, all were found to be positive for IgM. In these regards, the predictive value positive for measles surveillance was $100 \%$.

\section{Representativeness}

The measles surveillance report has been reported from the community level and the disease registries at health posts and health centers. However, the weekly surveillance reporting format lacks some important epidemiological variables like sex and age, which is vital to describe the distribution of diseases by personal characteristics and help to generate information for appropriate actions. Regarding the health service coverage, one health center and health post is expected to serve 25,000 and 5,000 individuals, respectively. A total of eight health centers and 32 health posts were available in the Ginnir district for the total population of 164,702. Therefore, the health service coverage in the district were above $100 \%$ by the health center and $97 \%$ by the health posts. Furthermore, the surveillance report was reported from all health facilities in the district.

\section{Stability}

Availability of the PHEM focal person both at the district and health facility level enabled the surveillance system to be operational with a limited resource. Of the 15 respondents interviewed, $12(80 \%)$ explained that shortage of budget and logistics specific to the surveillance system, and staff turnover were the common factors affecting the stability and well-functioning of the system to achieve its intended objectives. However, the surveillance system was integrated into the routine healthcare delivery and operational throughout the year.

\section{Discussion}

Public health surveillance systems need to be evaluated periodically to determine how well they operate, and the evaluation should emphasize attributes that are most important for the objectives of the system. ${ }^{1}$ We evaluated the performance of the measles surveillance system in Ginnir district, Southeast Ethiopia.

The surveillance system is fully integrated into the routine healthcare delivery system and have been implemented throughout the year. The community and health facilities in the districts were the main sources of information. The structure of data flow was from the community at the bottom to the respective upper level. The flow of data was well organized and the reporting facilities are familiar with the line of communication. Nevertheless, the flow of surveillance reporting and communication have several challenges, such as lack of wired telephone and internet access for regular communication.

Effective public health responses depend on the ability of the surveillance system to provide reliable, timely, and complete information to support action. ${ }^{10,12}$ According to the findings of our study, the weekly surveillance report completeness of health centers and health posts were $93 \%$ and $95 \%$, respectively, in 2018. Similarly, the overall report completeness of the district was $95 \%$. The report completeness in our finding is higher than the $90 \%$ national target set by the country by the year $2019 / 20,{ }^{13}$ and the target of $80 \%$ set by the World Health Organization. ${ }^{10}$ Our finding is also consistent with evaluation of a public health surveillance system in Dangila district, Northwest Ethiopia which reported a surveillance report completeness higher than the national target. ${ }^{14}$ This indicates acceptance of the public health surveillance system by all stakeholders engaged in the system.

The average surveillance report timeliness by the district was $87 \%$, which is higher than the target of $80 \%$ set by the World Health Organization, ${ }^{10}$ and the $61 \%$ report timeliness from a study conducted in Northern Ghana. ${ }^{15}$ However, the report timeliness in our study is lower than the $95 \%$ national target set nationally by the health sector transformation plan in $2019 / 20,{ }^{13}$ and the $94.6 \%$ report timeliness reported from a study conducted in Dangila 
district, Northwest Ethiopia. ${ }^{14}$ In addition, the district health office was not timely notified of the measles outbreak which occurred in 2019 to zonal health department, and the intervention activities to control the outbreak were also initiated late. Effective communication channel and timely reporting of surveillance data is required to provide timely information, which will help in early detection of health-related events and immediate action. ${ }^{5,7,10}$ The lower report timeliness in the study area might be due to a lack of regular lines of communication between the reporting site and the higher level, as indicated by the absence of a wired office telephone service and internet access.

According to the national guidelines, surveillance data analysis and interpretation should be done weekly at each level where the data are collected, starting from the health facility to the national level. ${ }^{5}$ The health centers in Ginnir district aggregate the data they received from health posts and send it to the district health office every Monday in the afternoon. However, the data are analyzed on weekly, monthly, and quarterly bases, and described by time, place, and person, having denominators like total population disaggregated by sex, age, and kebeles, etc. Similarly, the district health office simply merges the aggregate reports received from the health centers and sends to the zonal health department without analysis. Furthermore, monitoring of accumulation of susceptible individuals for measles over a time period is crucial in prediction and prevention of the measles outbreak, ${ }^{12}$ however, not being monitored and analyzed by the district health office. Similar findings were reported from the study in Dangila district, Northwest Ethiopia. ${ }^{14}$ The surveillance data must be analyzed and provide key information for decision-making and public health action. The possible reasons for poor performance in analysis of surveillance data are skill gap in data analysis and management, weak supervision and feedback system, poor commitment, and lack of continuous capacity building through updated training.

The district health office has an Emergency Preparedness and Response Plan (EPRP) which was not supported by the budget and supplies to respond to the emergencies. The health facilities did not have a written EPRP and outbreak investigation checklist. The rapid response team and multisectoral epidemic management committee established in the district were not fully functional. Additionally, the epidemic management committee has no documented minutes of meetings, review of plans, or lessons learnt by conducting post-epidemic evaluation. Our finding is supported by the report of study in Dangila district, Northwest Ethiopia. ${ }^{14}$ According to the measles outbreak investigation conducted in Ginnir district of Ethiopia in 2019, the intervention activities to control the outbreak was initiated late by the district, after 1 month of the outbreak and the outbreak response immunization targeting children aged 6 months to 14 years was also initiated too late after 2 months. ${ }^{9}$ Poor preparedness and response to the public health emergencies have an adverse consequence for the community. The possible reason for poor functionality of the rapid response team and epidemic management committee might be the absence of capacity building, absence of ownership sense, and poor supervision from the officials at a higher level.

When an outbreak is detected, it should be investigated in order to identify the source or the cause, to manage the cases, and to control and prevent further spread of the outbreak. $^{12}$ Outbreaks occur when the accumulated number of susceptible individuals is greater than the critical number of susceptible individuals for a given population to sustain transmission. ${ }^{12}$ However, the district health office did not investigate the measles outbreak which affected 1,043 people, with five deaths, in 2019, ${ }^{9}$ using epidemiological design. A study conducted in Dangila district, Northwest Ethiopia reported similar findings. ${ }^{14}$ According to the district PHEM department, the main reason for not investigating the outbreak was lack of capacity and skill to conduct an investigation according to its procedure.

Regular supportive supervision and feedback is crucial to improve the performance of the surveillance system. The Ginnir district health office and health centers were conducting a supportive supervision activity in irregular and integrated ways, and not using the supervision checklist specific to assess the surveillance activities. Developing a regular epidemiological bulletin which shows the trends of diseases over time and disseminating the bulletin for the surveillance officials is essential to build a strong feedback mechanism. However, a written feedback system for the performed activities was not practiced in the district. The main reason for the weak feedback system in the district is due to the absence of analysis and interpretation of surveillance data, and dissemination for the health facilities. Similar findings were reported from a study conducted in Dangila district, Northwest Ethiopia, ${ }^{14}$ and a study in Akwa-Ibom state of Nigeria. ${ }^{16}$ 
Regarding the attributes, the surveillance system of the district was found to be useful, simple, acceptable, flexible, and representative. The quality of surveillance data was poor in recording the time period of the report and the date of report received and sent. The average surveillance report timeliness was also lower $(87 \%)$, with no timely notification of outbreak or initiation of intervention by the district. The stability and functioning of the system to achieve its intended purpose have been challenged by the shortage of budget and logistics, staff turnover, absence of update training for health workers, and poor supportive supervision and feedback mechanism. Our finding is in line with the reports of study conducted in Dangila district, Northwest Ethiopia. ${ }^{14}$

This study has clearly demonstrated the performance of measles surveillance system in Ginnir district; however, our study has some constraints. There might be socially desirable responses by the surveillance personnel's working within the public health surveillance due to fear of measures by the officials as a result of disclosing the actual situations. But, prior to the data collection we held discussion with the stakeholders and briefed in detail the objective of the assessment which was to evaluate the performance of the surveillance system rather than the individual's performances.

\section{Conclusions}

The surveillance system of the district found to be useful, simple, acceptable, flexible, and representative. Quality of data, timeliness, and stability of the surveillance system were attributes that require improvement to enhance performance of the system. Weekly report completeness was above the target, but the timeliness of the report was below the national target. The surveillance system is fully integrated into the routine healthcare delivery system and the structure of surveillance data flow was from the community at the bottom to the respective upper level. However, the district surveillance system has the following gaps; poor line of communication between surveillance levels, lack of regular surveillance data analysis and interpretation, absence of EPRP at health facility level, lack of budget, poorly functional rapid response team and epidemic management committee, failure to investigate the outbreak, and weak supportive supervision, and feedback system. Therefore, to strengthen the performance of the surveillance system in prevention and control of measles, building a strong line of communication, regular analysis, and interpretation of surveillance data, preparation of epidemiological bulletin and dissemination to the stakeholders and regular supportive supervision and feedback is recommended for the surveillance focal of the district. Furthermore, zonal health departments are required to make functional the RRT and epidemic management committee, allocate budget for surveillance activities, and provide capacity building trainings for the surveillance officials at the district and health facility level.

\section{Abbreviations}

CDC, Center for Disease Control and Prevention; CFR, case fatality rate; EPRP, emergency preparedness and response plan; HEWs, health extension workers; IDSR, integrated disease surveillance and response; $\mathrm{MCV}$, measle containing vaccine; PHEM, public health emergency management; PVP, predictive value positive; RRT, rapid response team; WHO, World Health Organization.

\section{Data Sharing Statement}

Data will be available upon request from the corresponding author.

\section{Ethics Approval and Informed Consent}

Ethical clearance was obtained from the Institutional Review Board (IRB) of Institute of Health, Jimma University. The study was conducted in accordance with the Declaration of Helsinki. The letter of support which was obtained from Epidemiology Department of Jimma University and Bale Zonal Health Department was submitted to the Ginnir District Health Office. Permission to use the data and conduct the study was taken from Ginnir district health office and health facilities. Written informed consent was obtained from each participant after the purpose of the study was explained. They were also informed that all the data obtained from them would be kept confidential and no individual data was included in the study.

\section{Acknowledgments}

We are thankful to Ethiopian Field Epidemiology and Laboratory Training Program and Epidemiology Department, Jimma University for their facilitation and support. We gratefully acknowledge Bale Zonal Health Department, Ginnir District Health Office, health facilities for their cooperation and participation to the successful completion of this study. We would like to extend our gratitude to Mr. Desta Hiko (Associate Professor of 
Epidemiology, Jimma University) for his encouragement and guidance. We also appreciate all PHEM personnel for their willingness and cooperation in giving us the data on the surveillance system.

\section{Author Contributions}

All authors made a substantial contribution to the work reported, whether that is in the conception, study design, execution, acquisition of data, analysis and interpretation, or in all these areas; took part in drafting, revising or critically reviewing the article; gave final approval of the version to be published; have agreed on the journal to which the article has been submitted; and agree to be accountable for all aspects of the work.

\section{Funding}

Funding was not received from any organization for this study.

\section{Disclosure}

The authors report no conflicts of interest in this work.

\section{References}

1. Centers for Disease Control and Prevention. Updated guidelines for evaluating public health surveillance systems: recommendations from the guidelines working group. MMWR. 2001;1-35.

2. Buehler JW, Rothman KJ, Greenland S. Modern Epidemiology. 2nd ed. Philadelphia, PA: Lippencott-Raven; 1998.

3. Teutsch S, Thacker S. Planning a public health surveillance system. Epidemiol Bull Pan Am Health Organ. 1995;16:1-6.
4. Thacker SB, Stroup DF. Future directions for comprehensive public health surveillance and health information systems in the United States. Am J Epidemiol. 1994;140(383-97):383-397. doi:10.1093/ oxfordjournals.aje.a117261

5. Ethiopian Public Health Institute. Public Health Emergency Management Guidelines for Ethiopia. 2012.

6. World health Organization. WHO-GAVI Joint Statement: Ethiopia Launches Measles Vaccine Second Dose on 11th February 2019. Addis Ababa, Ethiopia; 2019.

7. World Health Organization. Global Measles and Rubella Update November 2018. Geneva, Switzerland; 2018.

8. Ethiopia World Health Organization. Health Cluster Bulletin \#7. 2019.

9. Kalil FS, Gemeda DH, Bedaso MH, et al. Measles outbreak investigation in Ginnir district of Bale zone, Oromia region, Southeast Ethiopia, May 2019. Pan Afr Med J. 2020;36:20. doi:10.11604/ pamj.2020.36.20.21169

10. World Health Organization AFR REGION. Technical Guidelines for Integrated Disease Surveillance and Response in the African Region. Atlanta, Georgea: CDC; 2010.

11. Central Statistical Agency. 2007 Population and Housing Census of Ethiopia: Administrative Report. 2012.

12. Ethiopian Health and Nutrition Research Institute. Guideline on Measles Surveillance and Outbreak Management. 3rd ed. Addis Ababa, Ethiopia; 2012.

13. Ethiopia Ministry of Health. Health Sector Transformation Plan 2015/16 - 2019/20. Ethiopia; 2015.

14. Alemu T, Gutema H, Legesse S, et al. Evaluation of public health surveillance system performance in Dangila district, Northwest Ethiopia: a concurrent embedded mixed quantitative/qualitative facility-based cross-sectional study. BMC Public Health. 2019;19 (1):1343. doi:10.1186/s12889-019-7724-y

15. Adokiya MN, Awoonor-Williams JK, Beiersmann C, et al. Evaluation of the reporting completeness and timeliness of the integrated disease surveillance and response system in northern Ghana. Ghana Med J. 2016;50(1):3-8. doi:10.4314/gmj.v50i1.1

16. Motilewa O, Akwaowo CD, Ekanem AM. Assessment of implementation of integrated disease surveillance and response in Akwa Ibom State Nigeria. Ibom Med J. 2015;8(1):24-25.
Risk Management and Healthcare Policy

\section{Publish your work in this journal}

Risk Management and Healthcare Policy is an international, peerreviewed, open access journal focusing on all aspects of public health, policy, and preventative measures to promote good health and improve morbidity and mortality in the population. The journal welcomes submitted papers covering original research, basic science, clinical \& epidemiological studies, reviews and evaluations, guidelines, expert opinion and commentary, case reports and extended reports. The manuscript management system is completely online and includes a very quick and fair peer-review system, which is all easy to use. Visit http://www.dovepress.com/testimonials.php to read real quotes from published authors. 